EUROPEAN ORGANISATION FOR NUCLEAR RESEARCH

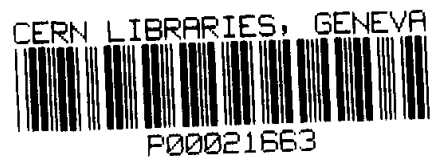

CERN-PPE/94-11

13.01 .94

Sw 9406

\title{
A Proton Tagging Detector for the NA48 Experiment
}

\author{
P. Grafström \\ CERN, Geneva, Switzerland
}

T. Beier, H. Blümer, T. Mehren, B. Renk, J. Staeck and O. Zeitnitz Institut für Physik, Universität Mainz, Mainz, Germany

M. Calvetti, P. Cenci, M. Pepe, M. Punturo, M.G. Setzu* and C. Talamonti Dipartimento di Fisica, Università degli Studi Di Perugia and INFN, Perugia, Italy

* Dipartimento di Fisica, Università degli Studi Di Cagliari and INFN, Cagliari, Italy

A. Taurok

Institut für Hochenergiephysik, Österreichische Akademie der Wissenschaften, Vienna, Austria

(Submitted to NIM)

\begin{abstract}
Prototype test results are reported from the NA48 $K_{S}$ tagging system. Thin scintillator foils were arranged as two staircases intersecting in space. A geometric alignment to $0.4 \mathrm{mrad}$, a light yield of 300-400 photoelectrons and a time resolution of 70-90 ps with conventional TDCs were achieved. A FADC system operating at 450 megasamples/s performed at $50 \mathrm{ps}$ intrinsic time resolution. Overlapping pulses could be resolved down to 7 ns.
\end{abstract}




\section{Introduction}

The NA48 experiment [1] at the CERN SPS aims at a measurement of the CP violation parameter $\operatorname{Re}^{\varepsilon^{\prime}} / \varepsilon$ to an overall accuracy of $2 \times 10^{-4}$. To achieve this the relative decay rates of short- and long-lived neutral kaons into two neutral and two charged pions are compared,

$$
\operatorname{Re}^{\varepsilon^{\prime} / \varepsilon}=\frac{1}{6}\left(1-\frac{\Gamma\left(K_{L} \rightarrow \pi^{0} \pi^{0}\right)}{\Gamma\left(K_{S} \rightarrow \pi^{0} \pi^{0}\right)} / \frac{\Gamma\left(K_{L} \rightarrow \pi^{+} \pi^{-}\right)}{\Gamma\left(K_{S} \rightarrow \pi^{+} \pi^{-}\right)}\right)
$$

Nearly collinear, overlapping beams will be used, where $K_{S}$ and $K_{L}$ are produced concurrently and distinguished by tagging the protons producing the $K_{S}$ component. Since the $K_{S}$ and $K_{L}$ beams coincide in space at the detector position, all detection efficiencies cancel to first order in the double ratio if the result is evaluated independently for different kaon momenta and decay positions. Accidental activities and the corresponding losses (gains) of good events, as well as time-dependant detector efficiencies, also cancel since all four decay modes are observed concurrently.

The $K_{S}$ or $K_{L}$ assignment will be done by measuring the time difference between the passage of a proton in the tagging counter upstream of the $K_{S}$ target and the event time in the detector. Events with a time difference inside a given interval $\Delta t$ will be called " $K_{S}$ ", any other events will be called " $K_{L}$ ". Figure 1 visualizes this concept which is equivalent to a time-of-flight measurement with a baseline of $250 \mathrm{~m}$.

Inefficiencies in the tagging counter such as misalignment and deadtime will cause $K_{S}$ decays to be identified as $K_{L}$, whereas accidental hits in the tagging counter will cause $K_{L}$ to $K_{S}$ transitions. These misidentifications are decay-mode independent. They lead to a correction of the double ratio but cannot generate an artificial non-zero value of $\operatorname{Re}^{\varepsilon^{\prime}} / \varepsilon$.

A primary proton beam of $\approx 10^{12}$ ppp is used to produce the $K_{L}$ beam at a small angle (K12 beam). After a first collimator the remaining protons emerging from the target are directed to a single, bent crystal [2]. This crystal deviates a small fraction of the primary protons by channeling and thus produces a $K_{S}$ proton beam of $\approx 3 \times 10^{7} \mathrm{ppp}$ which is passed through the tagging counter. Particular system requirements are

- good time resolution $\sigma_{t}<500$ ps to keep the time acceptance window $\Delta t$ for $K_{S}$ assignment small, e.g. $\pm 2.5 \mathrm{~ns}$;

- light material to preserve the quality of the proton beam;

- radiation hardness (1 SPS burst corresponds to $1 \mathrm{~Gy}$ );

- high efficiency; 
- high rate capability (>1 MHz) and virtually deadtime-less readout;

The operating conditions and the specifications listed above exclude the use of silicon or crystal counters. The present paper describes prototype tests of a scintillator tagging detector carried out during 1992 in the H6 beam at the CERN SPS.

\section{Prototype setup}

The final K12 proton beam of the NA48 experiment is expected to have an elliptical shape with flat particle area density and outer dimensions of about $3 \mathrm{~mm} \times 6 \mathrm{~mm}$. The tagging counter prototype was built from two sets of twelve staggered scintillators arranged alternatingly in horizontal and vertical orientation - see Fig. 2a. All scintillators were 4 mm thick along the beam direction $\left(0.01 \mathrm{X}_{0}\right)$ and had a transverse length of $15 \mathrm{~mm}$. NE102A scintillator [3] was chosen because of its suitable light emission characteristics, light yield and radiation hardness. To limit the individual counting rates to $1-2 \mathrm{MHz}$ the scintillators near the beam axis had a width of $200 \mu \mathrm{m}$ whereas those at the beam edges were up to $3000 \mu \mathrm{m}$ wide. In each projection successive scintillators had a nominal overlap of $50 \mu \mathrm{m}$ to ensure geometrical efficiency. On the left and right sides the scintillators were glued with their back surfaces on a rigid prototype brass support structure leaving a central beam passage of $8 \mathrm{~mm} \times 8 \mathrm{~mm}$ free of material except for the scintillators themselves - see Fig. $2 b$.

All back surfaces of the scintillators were coated with white paint [4] increasing the light output by a factor of two compared to aluminized mylar or thin $\mathrm{Al}$ coating. The scintillation light was collected with rectangular aluminized mylar envelopes through an air gap of $8 \mathrm{~mm}$ into lucite light guides and transmitted to the photomultipliers [5].

Anticounters were fixed on both sides of the structure to identify beam halo particles. Separate trigger counters were mounted in front of and behind the tagging counter.

Each light guide was equipped with an optical quartz fibre to transport ultra-violet light from a pulsed nitrogen laser [6] to the scintillators. This system provided monitoring pulses and was used to check the time resolution and light yield of the counters. The laser pulseheight was measured with a photodiode on a pulse-to-pulse basis. The spectra were corrected for intensity fluctuations and the relative width was then used to estimate the number of photoelectrons. Laser signals were lower than particle signals by $30 \%$. For laser pulses a typical time resolution of 90 ps per channel was achieved [7].

The light yield for beam particles was 300 to 400 photoelectrons. Landau tails in the pulseheight distributions were considerably suppressed in the thin scintillators due to 
scattering of $\delta$-electrons out of the foils. This effect is helpful in reducing the required dynamic range of the electronics to about 1:3.

To avoid geometrical inefficiencies the tagging counter must be positioned on the beam axis and aligned with a precision of better than $0.5 \mathrm{mrad}$. Three remote-controlled independent stepping motors allowed the entire counter to be moved vertically and to rotate along the vertical and the horizontal axes perpendicular to the beam. The vertical movement was used to scan the beam profile using the twelve horizontal scintillators. From a comparison of the actual and nominal beam positions the misalignment angle could be computed and corrected for to a precision of $0.4 \mathrm{mrad}$. A rotation around the vertical axis was used to align the vertically oriented scintillators. The multiplicity distribution and the number of counters hit by a single proton depend on the counter orientation with respect to the beam axis. Only the six central counters having equal width were used. From the number of events with one to five hits the overlap as a function of the angle could be obtained, while the number of six-hit events showed a marked peak when all counters were ligned up with the beam. The precision of the angular scan method was $0.2 \mathrm{mrad}$. The vertical and angular scans revealed that support structure and scintillators did not have the required mechanical tolerance of $\pm 5 \mu \mathrm{m}$. When the counters were aligned allowing for a larger overlap, however, the efficiency was consistent with $100 \%$. The final tagging counter will use a precise carbon fiber support structure [8].

\section{FADC}

Conventional electronics like discriminators and TDCs do not have the required double pulse separation and have large intrinsic dead-time. Instead, real-time pulseheight sampling at high rates is necessary. Therefore a Fast Analog-to-Digital Converter (FADC) was designed and four prototype modules with normal CAMAC readout were built (Fig. 3). Each module was equipped with a 6-bit FADC chip [9] running at sampling rates up to $500 \mathrm{MHz}$. From the FADC output the data are formatted into 48-bit words containing four pulseheight bytes plus timing information. A digital zerosuppression required at least three counts in any of these four successive samples. The input baseline could be shifted with a digital-to-analog converter to adjust the effect of the zero-suppression. A memory of 256 words of 48-bit data provided for on-board storage of pulsheight data and timing information over $2 \mu \mathrm{s}$. The data were latched to the CAMAC bus as three 16-bit words.

A block diagram of the electronics is shown in Fig. 4. Conventional electronics was used to measure the light yield, the time resolution and the efficiency at low rate. Photomultiplier signals were transmitted over $60 \mathrm{~m}$ of normal RG58 coaxial cable, amplified and fed into ADCs, TDCs and scalers.

Four selected channels could be connected to the FADC system which was started synchronously by a trigger counter. The time of a PM pulse was determined from the 
sampled pulseheights with a fit procedure. The convolution of a Gaussian smearing function of width $\sigma_{\mathrm{G}}$ with an exponential light pulse of decay time constant $\tau$ described the data well. First, the pulse shape parameters were optimized leaving $\sigma_{G}$ and $\tau$ free together with the time and the amplitude. Each channel required its own set of parameters, and the fit parameters depend on cable length, scintillator material and photomultiplier type as expected. Optimal values for $\sigma_{\mathrm{G}}$ and $\tau$ were obtained from a large number of non-overlapping pulses. Keeping the pulse shape parameters fixed, the program was then also used to determine the time and the amplitude of overlapping pulses.

The FADC performance was tested by recording the analog signals of the two trigger counters $\mathrm{T} 1$ and $\mathrm{T} 2$ at an average rate of $1.6 \mathrm{MHz}$. These counters measured $10 \times 10 \mathrm{~mm}^{2}$ and were built from $6 \mathrm{~mm}$ thick NE110 scintillator [3]. Figure 5 shows an example of overlapping pulses recorded with one of the trigger counters.

The intrinsic resolution of electronics and software was obtained from the measured time difference distribution of identical pulses split into different FADCs. The resolution is $70 \mathrm{ps}$, indicating individual contributions of $50 \mathrm{ps}$ per FADC. The main limitation was internal noise. The resolution is comparable to the performance of standard TDCs and meets our requirements well.

The time resolution of the central horizontal tagging counters obtained with two FADC is shown in Fig. 6a. The tagging counter was rotated so that the central horizontal counters $\mathrm{H} 4-\mathrm{H} 7$ were aligned on the beam axis and coincidences could be observed. Unfolding the FADC itself the contribution of each counter in the example is $(94 \pm 13)$ ps. Depending on operating conditions and event selection criteria resolutions down to $70 \mathrm{ps}$ per channel could be obtained.

Figure $6 \mathrm{~b}$ shows the distribution of waiting times between the first pulse in an event (which caused the trigger) and the second one (occurring randomly). Although statistics is limited the expected exponential shape is clearly visible. The first two bins are low because pulse distances below three sampling intervals are not resolved. For the 6-bit prototype running at $450 \mathrm{MHz}$ we therefore quote a double pulse resolving time of $7 \mathrm{~ns}$. This will be further improved by a higher sampling rate, low-noise 8-bit FADCs, shorter pulses and refined software.

\section{Conclusion}

The tagging component test has successfully demonstrated that the principal design criteria have been exceeded. A light yield of $>300$ photoelectrons, a counter time resolution of $<110 \mathrm{ps}$, an FADC time resolution of $50 \mathrm{ps}$ (6-bit, $450 \mathrm{MHz}$ ) and a double pulse separation of $<3$ sampling units have been obtained. Sensitive alignment procedures have been developed which allow the scintillators to be positioned to better 
than $0.5 \mathrm{mrad}$ with respect to the beam. The final FADCs will exploit the large area of the VXI D-size crate standard and use two 8-bit chips [10] running at $500 \mathrm{MHz}$ to obtain an effective sampling rate of $1 \mathrm{GHz}$. The readout will be virtually deadtimeless and fully pipelined into an optical fibre transmitter.

\section{Acknowledgements}

We wish to thank our technical staff A. Alaimo, E. Babucci, M. Bergauer, K. Fuchs, K.-H. Geib, E. Papi, P. Reuther and W. Schüller for their dedicated and skilful work. 


\section{Figure Captions}

Fig. 1: Concept of $K_{S}$ proton tagging in NA48.

Fig. 2 (a) Geometry of the tagging counter.

(b) Picture of the prototype brass support structure.

Fig. 3: FADC block diagram.

Fig. 4: Electronics block diagram.

Fig. 5: Overlapping pulses in one of the trigger counters (NE 110). The histogram represents the data with the fit result superimposed as a solid line.

Fig. 6 (a) Measured time differences between two individual counters. The tagging counter was rotated so that the central horizontal counters $\mathrm{H} 4-\mathrm{H} 7$ were aligned on the beam axis and coincidences could be observed. The resolution is $(150 \pm 9)$ ps including contributions from both photomultipliers and both FADCs. Unfolding the FADC contributions of 50 ps each the resolution of each counter is ( $94 \pm 13$ ) ps.

(b) Exponential distribution of waiting times from a given trigger to the next random event recorded with trigger counter T1 (NE110 scintillator). The slope gives the average rate of $1.6 \mathrm{MHz}$, consistent with direct rate measurements. The first two bins are low because such small pulse distances are not resolved, equivalent to a double pulse separation down to 3 sampling intervals or $7 \mathrm{~ns}$. 


\section{References}

[1] G. D. Barr et al., Proposal of the NA48 collaboration, CERN/SPSC/90-22, SPSC/P253, 20 July 1990.

[2] A.F. Elisheve et al., Phys. Lett. B88 (1979) 387; J.F.Bak et al., Phys. Lett B93 (1980) 505; M.Clement, N.Doble, L.Gatignon and P.Grafström, CERN/SL/92$21,1992$.

[3] Nuclear Enterprise, Edinburgh, Scotland. The factory quotes decay time constants of $2.4 \mathrm{~ns}$ for NE102A and $3.3 \mathrm{~ns}$ for NE110.

[4] BC-620 reflective paint, BICRON Corporation, Newbury, Ohio, USA.

[5] Hamamatsu Photonics. We used R2076 with voltage divider E2253-01.

[6] LTB Laser Technology Berlin, Berlin, Germany, model MSG 800-TD. Main features were $400 \mu \mathrm{J}$ pulse energy, $\leq 10 \mathrm{~Hz}$ repetition rate, $337 \mathrm{~nm}$ wavelenght and 500 ps FWHM pulse duration.

[7] LeCroy Research Systems, New York, USA: We used time-to-charge converters TFC 4303 and FERA ADCs $4300 \mathrm{~B} / 610$ with $40 \mathrm{ps} /$ count.

[8] Stesalit AG Kunstoffwerk, CH-4234 Zullwil, Switzerland.

[9] AD 9016P from Analog Devices, Norwood, Massachusets, USA.

[10] TKAD10C from Tektronix U.K. Ltd., London, United Kingdom. 


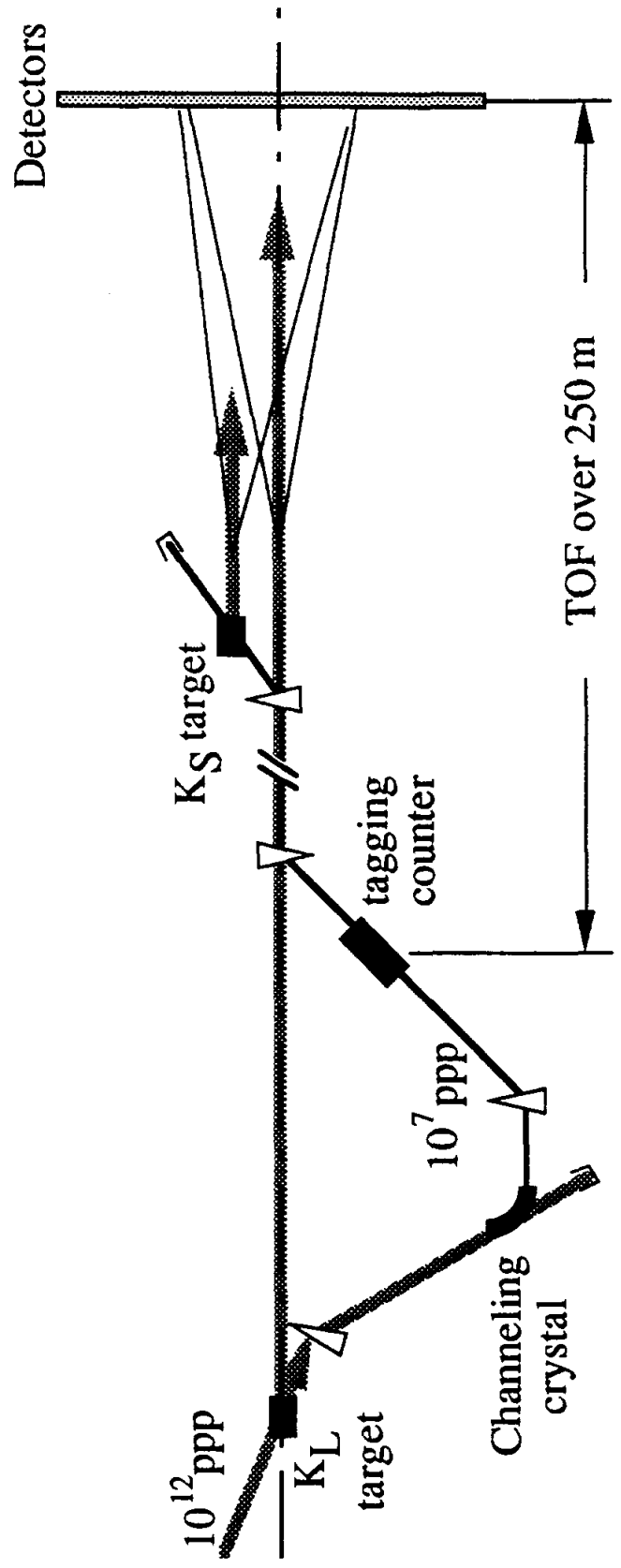

Figure 1 


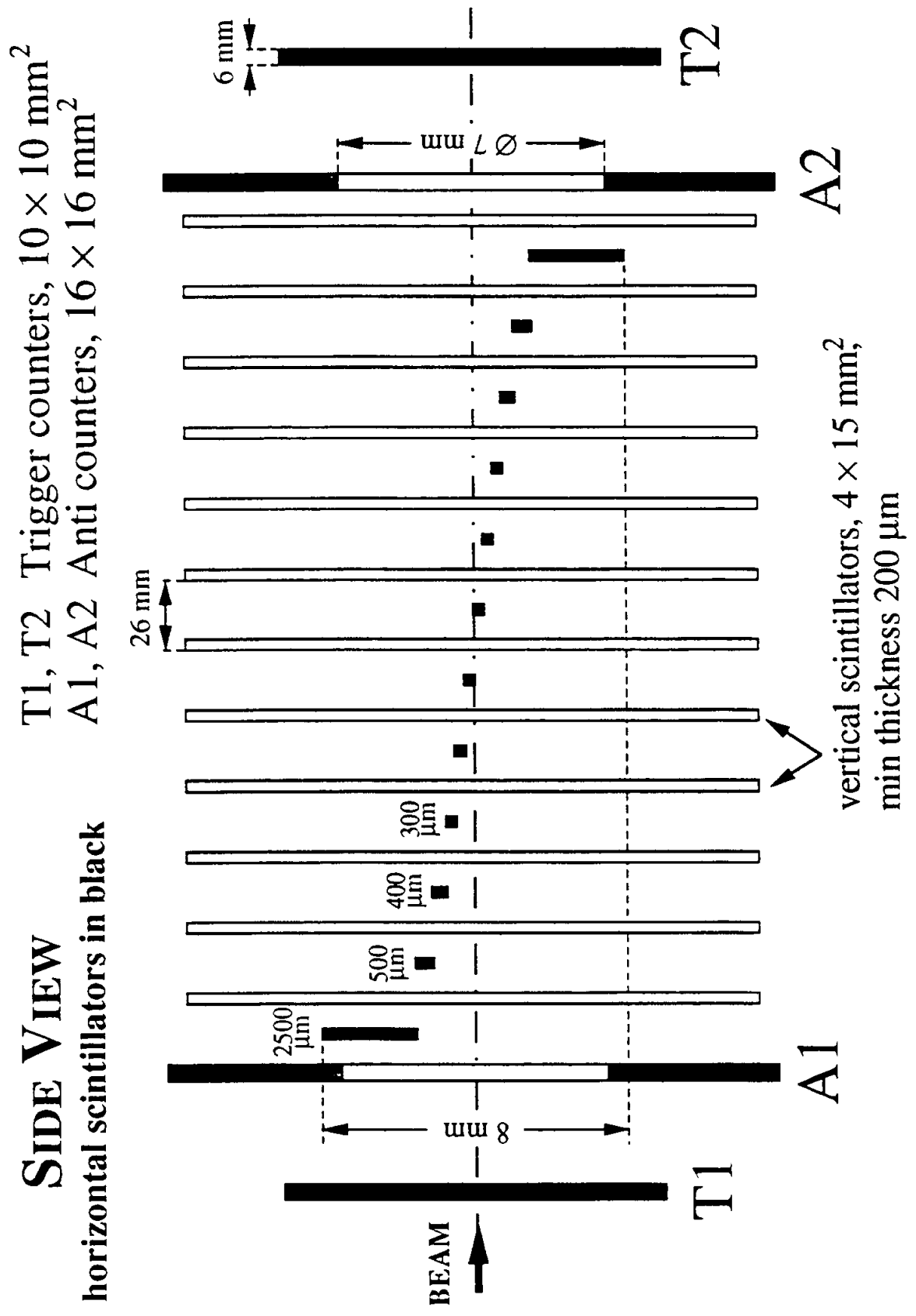

Figure 2a 


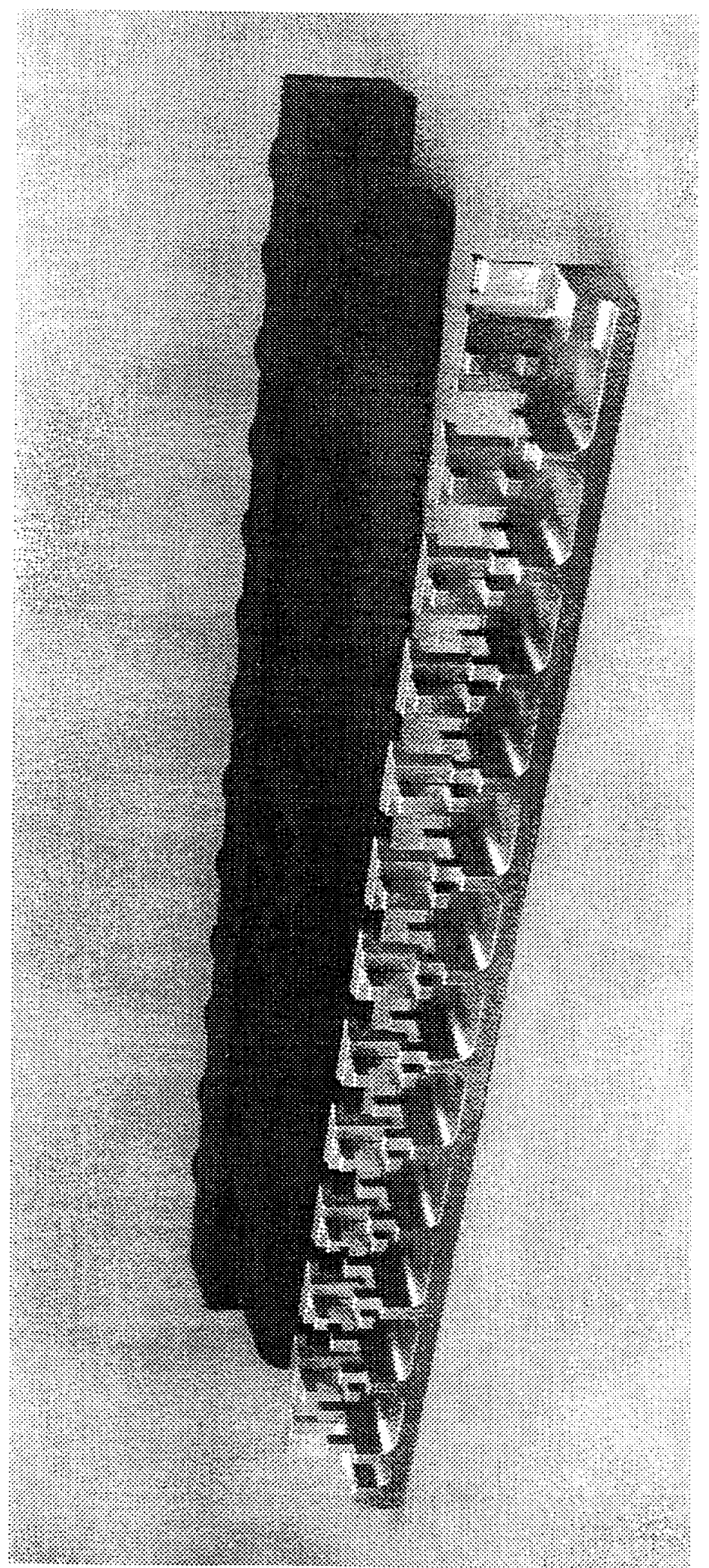

Figure 2b 


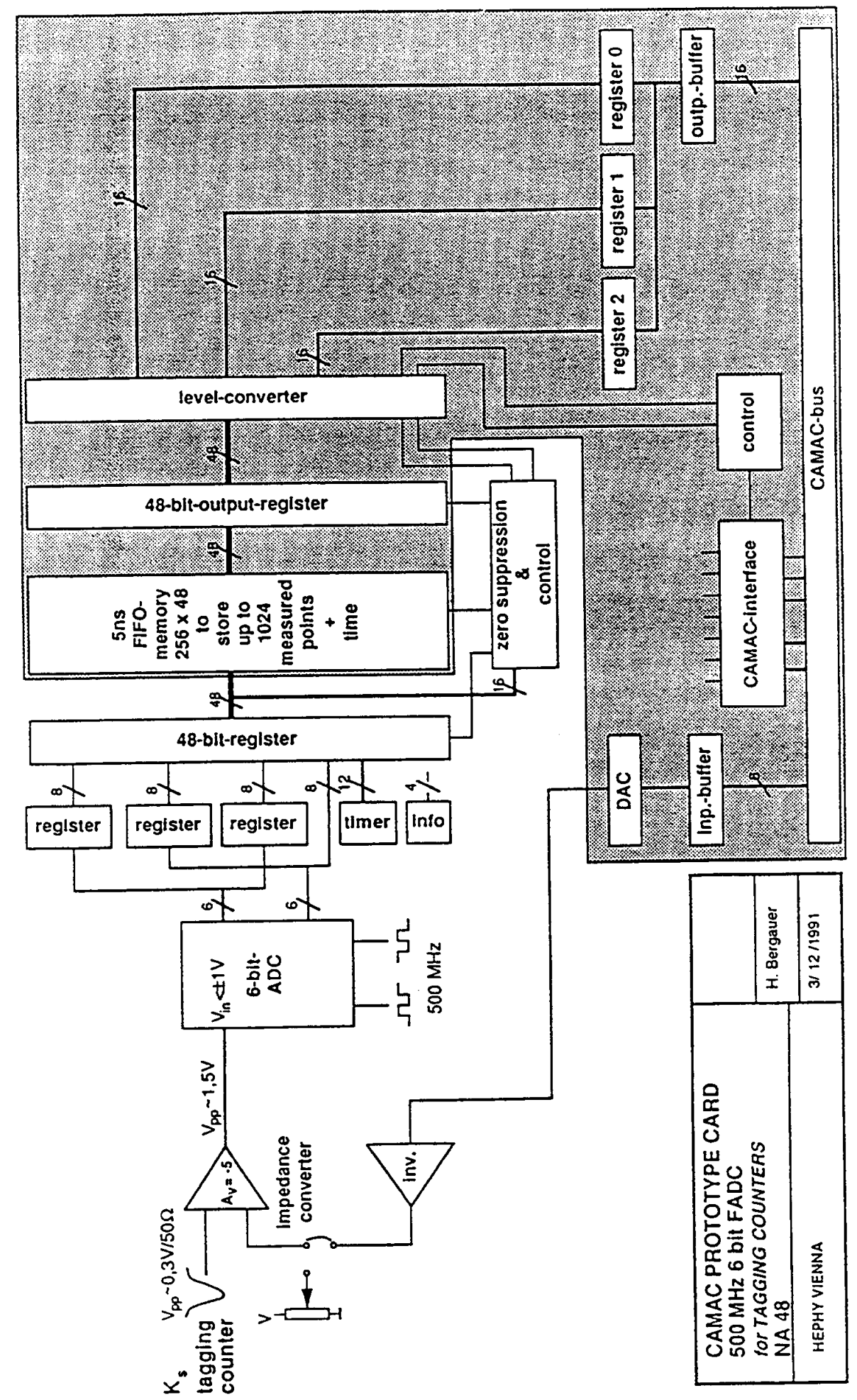

Figure 3 


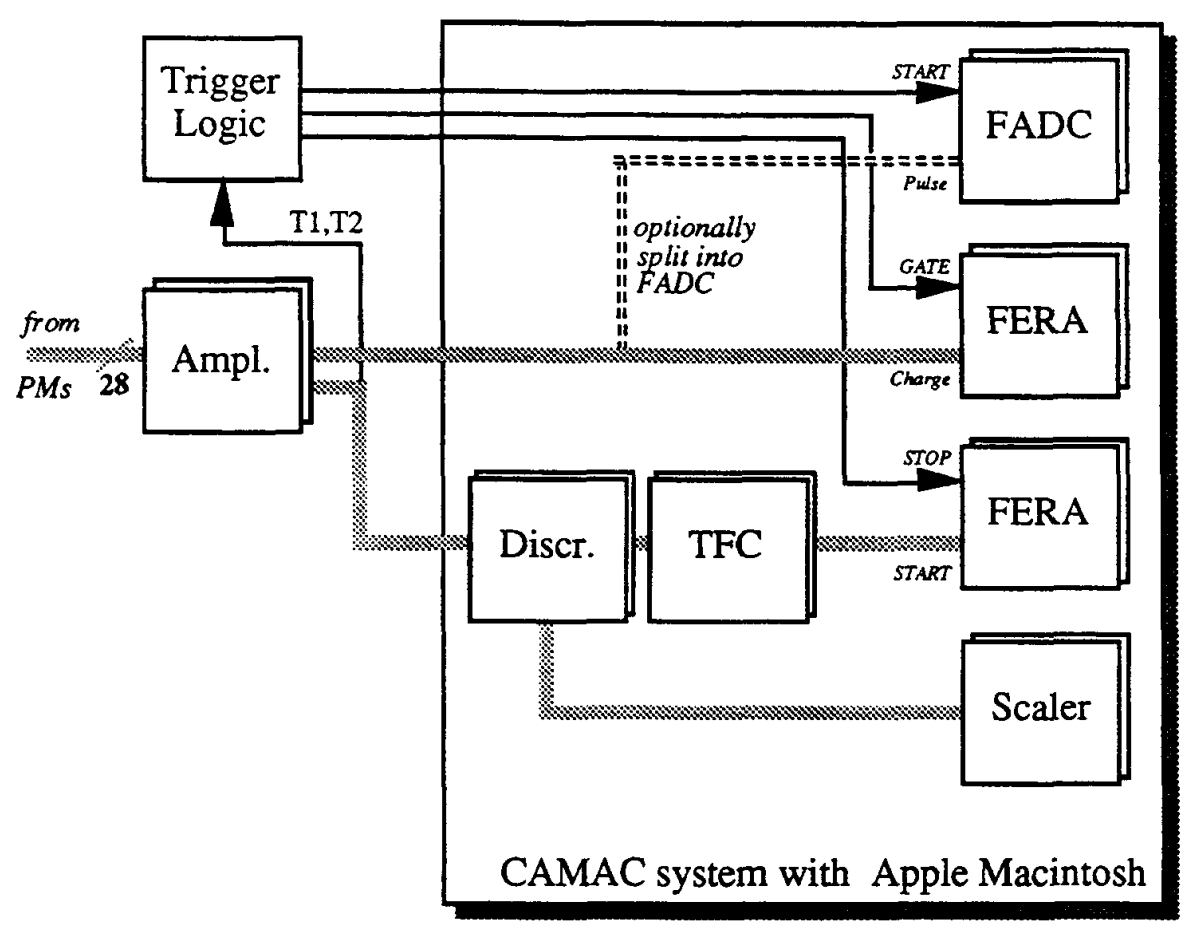

Figure 4 


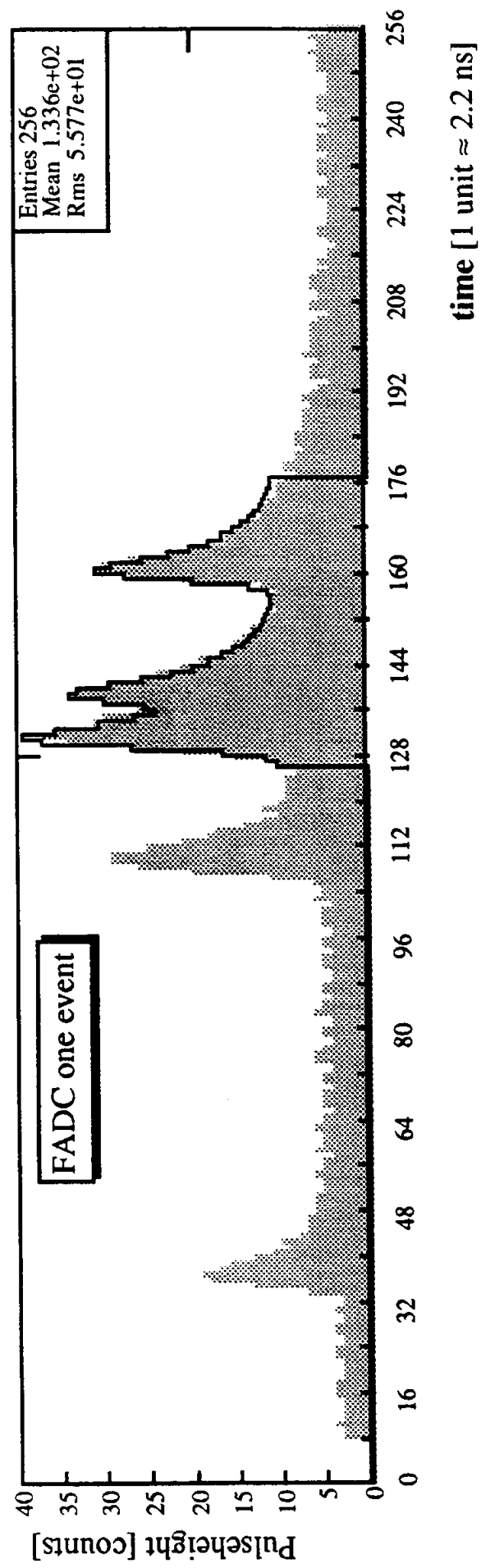

Figure 5 


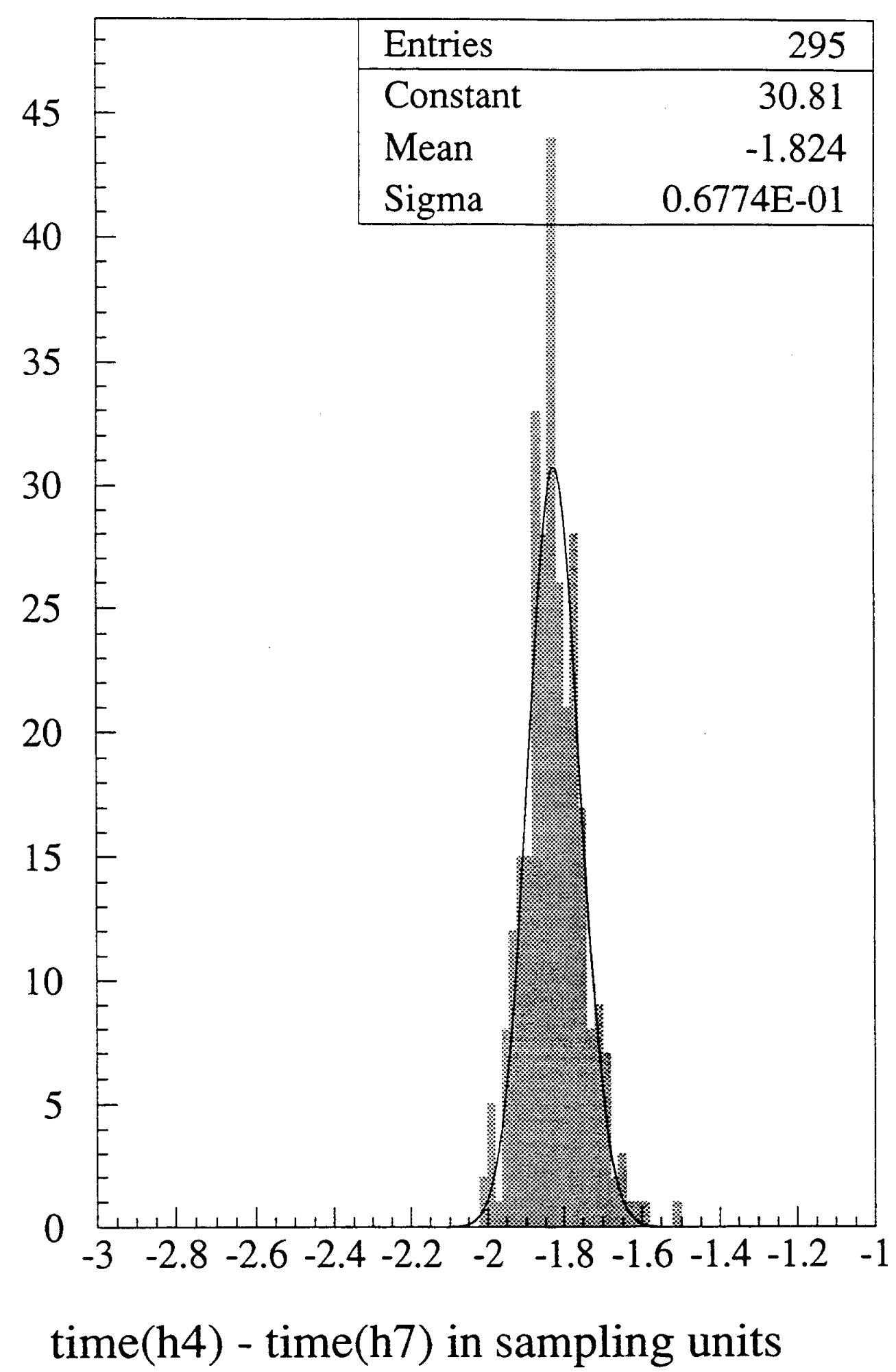

Figure 6a 


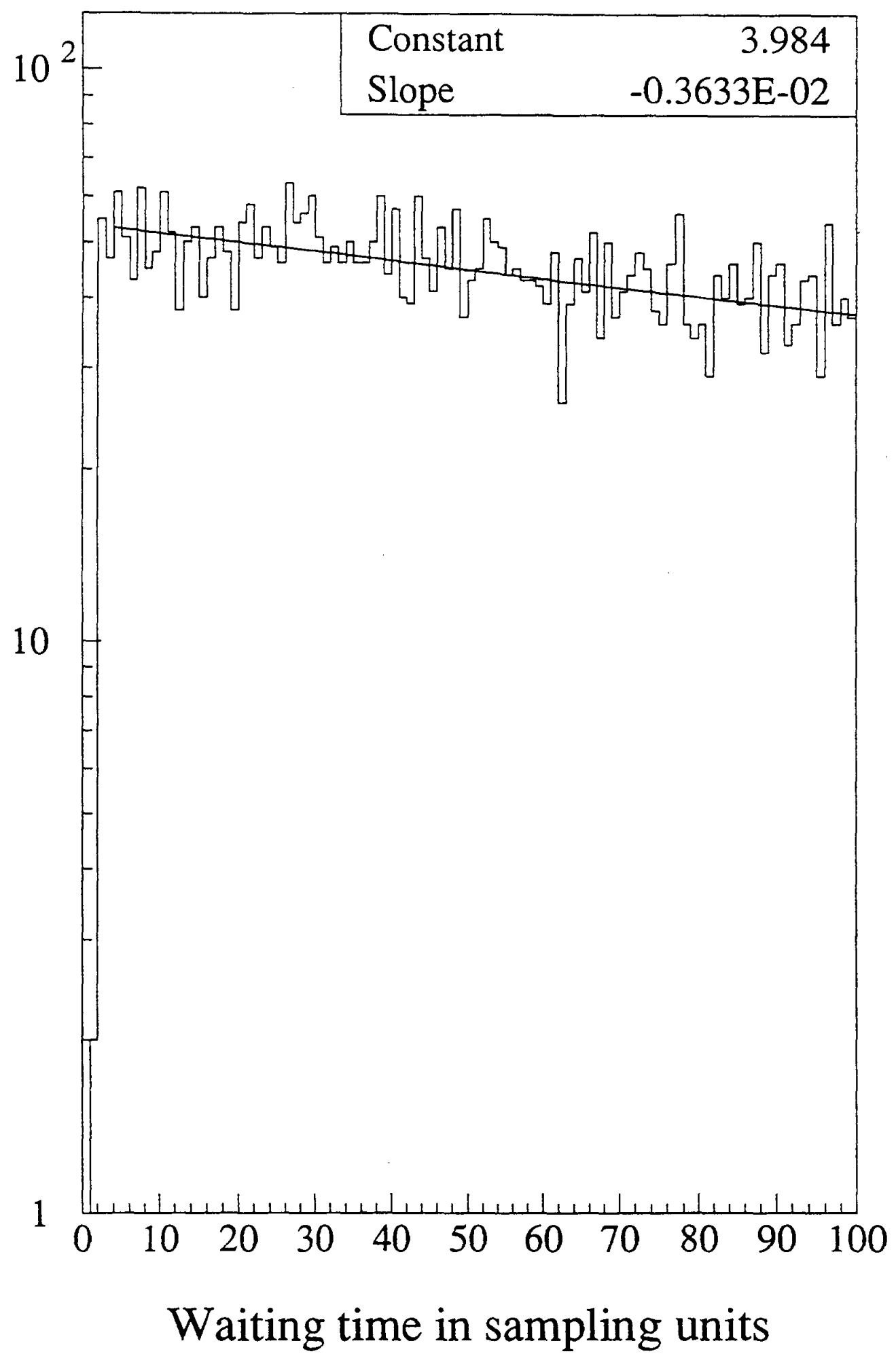

Figure 6b 